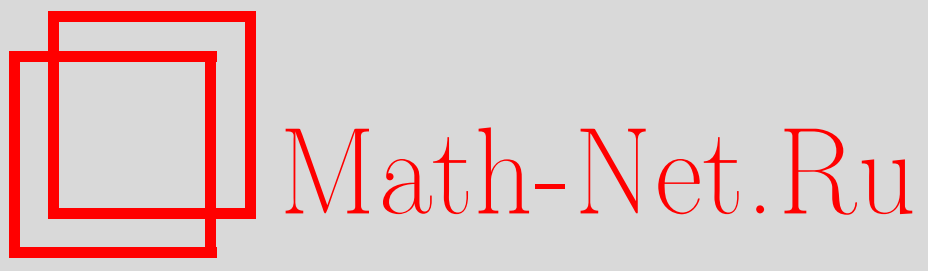

С. Б. Куксин, А. Р. Ширикян, О случайных аттракторах для систем перемешивающего типа, Функи. анализ и его прил., 2004, том 38, выпуск 1, 34-46

DOI: https://doi.org/10.4213/faa94

Использование Общероссийского математического портала MathNet.Ru подразумевает, что вы прочитали и согласны с пользовательским соглашением

http: //www . mathnet.ru/rus/agreement

Параметры загрузки:

IP : 35.173 .219 .12

26 апреля 2023 г., 15:47:32

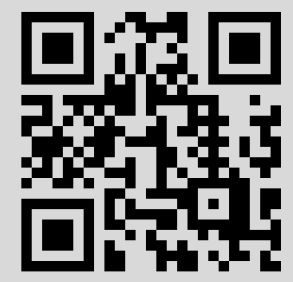


Функииональньй анализ и его приложения

2004, т. 38, вып. 1, с. 34-46

УДК $517.95+517.946$

\title{
О случайных аттракторах для систем перемешивающего типа*
}

\author{
(c) 2004. С. Б. Куксин, А. Ширикян
}

Посвящается И. М. Гельфанду к его 90-летию

\section{Введение}

Статья посвящена случайным динамическим системам (СДС)

$$
\varphi_{k}: H \rightarrow H, \quad H \ni u \mapsto \varphi_{k} u, \quad k \geqslant 0,
$$

на польском ${ }^{1)}$ пространстве $H$. Здесь $\varphi_{k}-$ случайное преобразование (так что $\varphi_{k}=\varphi_{k}(\omega)$, где $\omega-$ случайный параметр). Предполагается, что как функция от $k$ это преобразование имеет независимые приращения. Как правило, время будет считаться дискретным (т.е. $k \in \mathbb{Z}_{+}$), однако мы кратко обсудим СДС с непрерывным временем, когда будем обсуждать стохастические уравнения с частными производными.

В значительной степени информация о долговременном поведении траекторий системы (0.1) содержится в свойствах ее случайного аттрактора. Среди многих возможных определений случайного аттрактора (см., например, $[3,5,6])$ мы выбираем следующее: компактное случайное множество $\mathscr{A}_{\omega}$ называется $c л y$ чайным аттрактором системы, если все траектории $\varphi_{k}(\omega) u$ этой системы сходятся к $\mathscr{A}_{\omega}$ по вероятности. По поводу точного определения и его обсуждения см. разд. 1.2.

СДС (0.1) определяет марковскую цепь в пространстве $H$ с переходной функцией

$$
P_{k}(u, \Gamma)=\mathbf{P}\left\{\omega: \varphi_{k}(\omega) u \in \Gamma\right\},
$$

где $\Gamma$ - борелевское подмножество пространства $H$. Долговременное поведение этого случайного процесса в существенной степени описывается его стационарными мерами. Напомним, что вероятностная мера $\mu$ на $H$ называется стационарной для марковской цепи с переходной функцией $(0.2)$, если $\mu(\Gamma)=$ $\int_{H} P_{k}(u, \Gamma) \mu(d u)$ для любого $k \geqslant 0$ и любого борелевского множества $\Gamma \subset H$. Для обсуждаемого класса систем всякая стационарная мера $\mu$ допускает марковскую дезинтеграцию:

$$
\mu(\Gamma)=\mathbf{E} \mu_{\omega}(\Gamma) .
$$

Здесь $\omega \mapsto \mu_{\omega}$ является мерозначным отображением, измеримым относительно прошлого, т. е. относительно сигма-алгебры, порожденной случайными преобразованиями $\varphi_{k}\left(\theta_{-m} \omega\right), k \geqslant m \geqslant 0$, где $\left\{\theta_{n}\right\}-$ соответствующие измеримые

*Эта работа была поддержана EPSRC, грант GR/N63055/01.

1) Польским называется полное сепарабельное метрическое пространство. 
преобразования вероятностного пространства, сохраняющие меру Р. См. [3,6] и разд. 1.1. Известно, что

$$
\operatorname{supp} \mu_{\omega} \subset \mathscr{A}_{\omega} \quad \text { п. н., }
$$

где $\mathscr{A}_{\omega}$ - произвольный случайный аттрактор, см. [7] и разд. 1.2.

Основным результатом этой статьи является теорема 2.4, которая утверждает, что носитель дезинтеграции единственной стационарной меры СДС (0.1) является ее минимальным случайным аттрактором, если система удовлетворяет некоторому неограничительному условию компактности и является системой перемешивающего типа в следующем смысле: для любой непрерывной ограниченной функции $f: H \rightarrow \mathbb{R}$

$$
\mathbf{E} f\left(\varphi_{k}(\omega) u\right) \rightarrow \int_{H} f(u) \mu(d u) \text { при } k \rightarrow+\infty,
$$

где $u \in H-$ произвольная начальная точка и $\mu-$ стационарная мера. Другими словами, при наложенных выше ограничениях соотношение $(0.3)$, где $\mathscr{A}_{\omega}-$ минимальный случайный аттрактор, становится равенством. Доказательство основано на эргодической теореме для динамической системы $\Theta_{k}$ на фазовом пространстве $\Omega \times H$, равной косому произведению преобразований $\theta_{k}$ и $\varphi_{k}$ :

$$
\Theta_{k}(\omega, u)=\left(\theta_{k} \omega, \varphi_{k}(\omega) u\right), \quad k \geqslant 0,
$$

см. теорему 2.3.

В $§ 4$ мы рассматриваем случайно возмущенную двумерную систему НавьеCтокса

$$
\dot{u}-\nu \Delta u+(u, \nabla) u+\nabla p=\eta(t, x), \quad \operatorname{div} u=0,
$$

где $u=u(t, x)$ - поле скорости жидкости, $p=p(t, x)$ - давление и $\eta(t, x)$ - случайная внешняя сила. Уравнения дополняются краевыми условиями Дирихле либо периодическими краевыми условиями. Случайная сила $\eta$ является гладкой по $x$, в то время как по $t$ она является либо толчковой силой, т. е. случайной дельта-мерой, сосредоточенной в целых моментах времени (в этом случае (0.5) определяет СДС с дискретным временем), либо белым шумом (в этом случае (0.5) определяет СДС с непрерывным временем). В обоих случаях полученная СДС удовлетворяет условию компактности. Предполагая выполненным некоторое неограничительное условие невырожденности, мы извлекаем из работ $[13,14]$, что эта СДС также удовлетворяет требуемому условию типа перемешивания. Таким образом, абстрактные теоремы 2.3 и 2.4 применимы к системе (0.5) как в случае толчковой, так и в случае белой силы. Соответственно носитель марковской дезинтеграции единственной стационарной меры является минимальным случайным аттрактором для (0.5) (см. теоремы 4.1 и $4.2)$, и функционалы, зависящие как от решения $u$, так и от соответствующей силы, удовлетворяют теореме эргодического типа (см. теорему 4.3).

Обозначения. Через $(H, d)$ обозначается польское пространство, через $C_{b}(H)$ - пространство ограниченных непрерывных функций на $H$, наделенное супремум-нормой $\sup _{u \in H}|f(u)|$, и через $L(H)$ - пространство функций $f: H \rightarrow \mathbb{R}$, таких, что

$$
\|f\|_{L(H)}=\sup _{u \in H}|f(u)|+\sup _{u, v \in H} \frac{|f(u)-f(v)|}{|u-v|}<\infty .
$$


Если $(\Omega, \mathscr{F}, \mathbf{P})$ - вероятностное пространство и $\mathscr{F}^{\prime}-$ произвольная сигмаподалгебра в $\mathscr{F}$, то через $\mathbb{L}\left(H, \mathscr{F}^{\prime}\right)$ мы обозначаем множество функций $F(\omega, u)$ : $\Omega \times H \rightarrow \mathbb{R}$, которые $\mathscr{F}^{\prime}$-измеримы по $\omega$ для каждого $u \in H$ и удовлетворяют условию

$$
\operatorname{ess} \sup _{\omega \in \Omega}\|F(\omega, \cdot)\|_{L(H)}<\infty .
$$

Для $u \in H$ и $A \subset H$ мы определяем расстояние между $u$ и $A$ формулой

$$
d(u, A)=\inf _{v \in A} d(u, v) .
$$

Благодарности. Мы признательны Дж. Робинсону за полезные обсуждения.

\section{§1. Предварительные результаты}

В этом параграфе мы напомним основные понятия из теории СДС и сформулируем некоторые вспомогательные результаты. В основном мы следуем книге [3]. Для упрощения изложения мы ограничимся системами с дискретным временем.

1.1. Случайные динамические системы. Пусть $(\Omega, \mathscr{F}, \mathbf{P})$ - вероятностное пространство, $\theta_{k}: \Omega \rightarrow \Omega, k \in \mathbb{Z},-$ группа сохраняющих меру автоморфизмов пространства $\Omega$ и $H$ - польское пространство, наделенное метрикой $d$ и борелевской $\sigma$-алгеброй $\mathscr{B}_{H}$.

ОПРЕДЕЛЕНИЕ 1.1. (Непреръвная) случайная динамическая система над $\theta_{k}$ определяется как семейство преобразований $\varphi_{k}(\omega): H \rightarrow H$, где $k \in \mathbb{Z}_{+}$и $\omega \in \Omega$, обладающее следующими свойствами:

(i) Измеримость. Отображение $(\omega, u) \mapsto \varphi_{k}(\omega)$, отображающее пространство $\Omega \times H$, наделенное сигма-алгеброй $\mathscr{F} \otimes \mathscr{B}_{H}$, в $H$, измеримо для любого $k \geqslant 0$.

(ii) Непрерывность. Для любых $\omega \in \Omega$ и $k \geqslant 0$ отображение $\varphi_{k}(\omega)$ непрерывHO.

(iii) Свойство коцикла. Для любых $\omega \in \Omega$ мы имеем

$$
\varphi_{0}(\omega)=\operatorname{Id}_{H}, \quad \varphi_{k+l}(\omega)=\varphi_{k}\left(\theta_{l} \omega\right) \circ \varphi_{l}(\omega) \quad \text { для всех } k, l \geqslant 0 .
$$

Для любых целых $m \leqslant n$ мы обозначаем через $\mathscr{F}[m, n]$ сигма-алгебру, порожденную $H$-значными случайными величинами $\varphi_{k}\left(\theta_{m-1} \omega\right) u$, где $u \in H$ и $k=0, \ldots, n-m+1$. Мы распространим это обозначение на случай $m=-\infty$ и/или $n=+\infty$, полагая $\mathscr{F}_{[-\infty, n]}=\sigma\left\{\mathscr{F}_{[m, n]}, m \leqslant n\right\}$ и т. д. Прямые вычисления показывают, что

$$
\theta_{k}^{-1} \mathscr{F}_{[m, n]}=\mathscr{F}_{[m+k, n+k]} \text { для всех } m \leqslant n \text { и } k .
$$

Сигма-алгебры $\mathscr{F}^{-}=\mathscr{F}_{[-\infty, 0]}$ и $\mathscr{F}^{+}=\mathscr{F}_{[1,+\infty]}$ называются прошлым и будущuм СДС $\varphi_{k}(\omega)$.

Пусть $\mathscr{P}_{\mathbf{P}}$ - семейство вероятностных мер на $\left(\Omega \times H, \mathscr{F} \otimes \mathscr{B}_{H}\right)$, таких, что их проекции на $\Omega$ совпадают с P. Хорошо известно (см. $[3$, Sec. 1.4]), что произвольная мера $\mathfrak{M} \in \mathscr{P}_{\mathbf{P}}$ имеет единственную дезинтеграцию $\omega \mapsto \mu_{\omega}$, являющуюся случайной величиной в пространстве мер, такой, что

$$
\mathfrak{M}(\Gamma)=\int_{\Omega} \int_{H} I_{\Gamma}(\omega, u) \mu_{\omega}(d u) \mathbf{P}(d \omega) \quad \forall \Gamma \in \mathscr{F} \otimes \mathscr{B}_{H},
$$


где $I_{\Gamma}$ обозначает индикаторную функцию множества $Г$.

Исходя из произвольной СДС $\varphi_{k}(\omega)$ над $\theta_{k}$, определим следующую полугруппу измеримых преобразований пространства $\Omega \times H$ :

$$
\Theta_{k}(\omega, u)=\left(\theta_{k} \omega, \varphi_{k}(\omega) u\right), \quad k \geqslant 0 .
$$

Полугруппа $\Theta_{k}$ называется косым произведением группы $\theta_{k}$ и динамической системы $\varphi_{k}(\omega)$. Мера $\mathfrak{M} \in \mathscr{P}_{\mathbf{P}}$ называется инвариантной относительно $\Theta_{k}$, если $\Theta_{k} \mathfrak{M}=\mathfrak{M}\left(\right.$ т. е. $\mathfrak{M}\left(\Theta_{k}^{-1}(\Gamma)\right)=\mathfrak{M}(\Gamma)$ для любого $\left.\Gamma \in \mathscr{F} \otimes \mathscr{B}_{H}\right)$. По теореме 1.4 .5 в [3] мера $\mathfrak{M} \in \mathscr{P}_{\mathbf{P}}$ инвариантна тогда и только тогда, когда ее дезинтеграция $\mu_{\omega}$ удовлетворяет следующему соотношению для $\mathbf{P}$-п. в. $\omega \in \Omega$ :

$$
\varphi_{k}(\omega) \mu_{\omega}=\mu_{\theta_{k} \omega} \quad \text { для всех } k \geqslant 0 .
$$

Множество всех инвариантных относительно $\Theta_{k}$ мер будет обозначаться через $\mathscr{I}_{\mathbf{P}}(\varphi)$.

ОПРЕДЕЛЕНИЕ 1.2. Инвариантная мера $\mathfrak{M} \in \mathscr{I}_{\mathbf{P}}(\varphi)$ называется марковской, если ее дезинтеграция $\mu_{\omega}$ измерима относительно прошлого $\mathscr{F}^{-}$. Множество всех таких мер будет обозначаться через $\mathscr{I}_{\mathbf{P}, \mathscr{F}}(\varphi)$.

Теперь перейдем к важному классу СДС с независимыми приращениями (называемыми также СДС типа белого шума).

ОПРЕДЕЛЕНИЕ 1.3. Случайная динамическая система $\varphi_{k}(\omega)$ называется $c u-$ стемой с независимыми приращениями, если ее прошлое и будущее независимы.

В силу соотношений (1.2) СДС $\varphi_{k}(\omega)$ имеет независимые приращения тогда и только тогда, когда сигма-алгебры $\mathscr{F}[m, n]$ и $\mathscr{F}_{\left[m^{\prime}, n^{\prime}\right]}$ независимы для любых непересекающихся интервалов $[m, n]$ и $\left[m^{\prime}, n^{\prime}\right]$ (конечных либо бесконечных).

Для любой СДС $\varphi_{k}(\omega)$ с независимыми приращениями множество случайных последовательностей $\left\{\varphi_{k}(\cdot) u, k \geqslant 0\right\}, u \in H$, образует семейство марковских цепей по отношению к фильтрации $\mathscr{F}_{k}=\theta_{k}^{-1} \mathscr{F}^{-}$. Соответствующая переходная функция имеет вид (0.2), а марковские операторы задаются формулами

$$
\mathfrak{P}_{k} f(u)=\int_{H} P_{k}(u, d v) f(v), \quad \mathfrak{P}_{k}^{*} \mu(\Gamma)=\int_{H} P_{k}(u, \Gamma) \mu(d u),
$$

где $f \in C_{b}(H)$ и $\mu \in \mathscr{P}(H)$. Напомним, что мера $\mu \in \mathscr{P}(H)$ называется $c m a-$ иионарной мерой для этого марковского семейства, если $\mathfrak{P}_{1}^{*} \mu=\mu$. Множество таких мер будет обозначаться через $\mathscr{S}_{\varphi}$. Следующий важный результат получен (в разных контекстах) в работах $[6,15,16]$.

ПРЕДЛОЖЕНИЕ 1.4. Пусть $\varphi_{k}(\omega)$ есть СДС с независимыми приращениями. Тогда имеется взаимно однозначное соотношение межсу марковскими инвариантными относительно косого произведения $\Theta_{k}$ мерами $\mathscr{I}_{\mathbf{P}, \mathscr{F}}(\varphi)$ и стаицонарными мерами $\mathscr{S}_{\varphi}$ соответствующего марковского семейства. А именно, если $\mu \in \mathscr{S}_{\varphi}$, то предел

$$
\mu_{\omega}=\lim _{k \rightarrow+\infty} \varphi_{k}\left(\theta_{-k} \omega\right) \mu
$$

существует в *-слабой топологии почти наверное и задает дезинтеграцию марковской инвариантной меры $\mathfrak{M}$. Обратно, если $\mathfrak{M} \in \mathscr{I}_{\mathbf{P}, \mathscr{F}-}-$ марковская инвариантная мера и $\mu_{\omega}-$ ее дезинтеграция, то $\mu=\mathbf{E} \mu_{\omega}$ является стационарной мерой для марковского семейства. 
1.2. Точечные аттракторы. Пусть $\left\{\varphi_{k}(\omega)\right\}$ - СДС в польском пространстве $H$ над $\left\{\theta_{k}\right\}$, такая же, как выше. Семейство подмножеств $\mathscr{A}_{\omega}, \omega \in \Omega$, называется случайным компактным (замкнутым) множеством, если множество $\mathscr{A}_{\omega}$ компактно (замкнуто) для п. в. $\omega$ и $\Omega_{U}:=\left\{\omega \in \Omega: \mathscr{A}_{\omega} \cap U \neq \varnothing\right\} \in \mathscr{F}$ для любого открытого множества $U \subset H$. Случайное компактное множество $\mathscr{A}_{\omega}$ называется измеримым по отношению к сигма-подалгебре $\mathscr{F}^{\prime} \subset \mathscr{F}$, если $\Omega_{U} \in \mathscr{F}^{\prime}$ для любого открытого множества $U \subset H$.

ОПредЕЛЕНИЕ 1.5. Случайное компактное множество $\mathscr{A}_{\omega}$ называется случайным точечным аттрактором (в смысле сходимости по вероятности), если для любого $u \in H$ последовательность случайных величин $d\left(\varphi_{k}(\omega) u, \mathscr{A}_{\theta_{k} \omega}\right)$ сходится к нулю по вероятности, т. е. для любого $\delta>0$

$$
\lim _{k \rightarrow+\infty} \mathbf{P}\left\{d\left(\varphi_{k}(\omega) u, \mathscr{A}_{\theta_{k} \omega}\right)>\delta\right\}=0 .
$$

Случайный точечный аттрактор $\mathscr{A}_{\omega}$ называется минимальным, если для любого случайного точечного аттрактора $\mathscr{A}_{\omega}^{\prime}$ мы имеем $\mathscr{A}_{\omega} \subset \mathscr{A}_{\omega}^{\prime}$ для п. в. $\omega$.

Понятно, что минимальный случайный точечный аттрактор единствен (если он существует), т. е. если $\mathscr{A}_{\omega}$ и $\mathscr{A}_{\omega}^{\prime}-$ два минимальных случайных аттрактора, то $\mathscr{A}_{\omega}=\mathscr{A}_{\omega}^{\prime}$ п. н. Так как преобразования $\theta_{k}$ сохраняют меру, то равенство $(1.4)$ эквивалентно соотношению

$$
\lim _{k \rightarrow+\infty} d\left(\varphi_{k}\left(\theta_{-k} \omega\right) u, \mathscr{A}_{\omega}\right)=0,
$$

где предел понимается в смысле сходимости по вероятности. Подобный тип сходимости траекторий к случайному множеству - когда начальное условие задано в момент времени $-k, k \rightarrow \infty$, и расстояние измеряется в нулевой момент - обычно используется для определения случайных аттракторов. Мы предпочитаем «прямое» определение (1.4), которое кажется нам более естественным. Отметим, что среди многих различных типов случайных аттракторов, рассматриваемых в современной математической литературе, аттрактор из определения 1.5 является наименьшим, см. $[3,5,6]$.

Если в определении 1.5 заменить соотношение (1.4) на условие, состоящее в том, что сходимость (1.5) имеет место для всех $u \in H$ и $\omega \in \Omega_{0}$, где $\Omega_{0} \in \mathscr{F}$ - событие полной меры, не зависящее от $u$, то получится определение случайного точечного аттрактора в смысле сходимости почти наверное). Так как п. н.-сходимость влечет за собой сходимость по вероятности, то такой аттрактор также удовлетворяет условию (1.4). В дальнейшем в основном рассматриваются случайные точечные аттракторы в смысле сходимости по вероятности, которые для краткости называются случайными аттракторами.

Следующий результат является непосредственным следствием теорем 3.4, 4.3 и замечания 3.5 (iii) из [7].

ПредЛОЖЕНИЕ 1.6. (i) Пусть $\varphi_{k}(\omega)$ есть СДС с независимыми приращениями. Предположим, что существует случайное компактное множество $\mathscr{K}_{\omega}$, притягивающее траектории системы $\varphi_{k}(\omega)$ в следующем смысле: найдется множество $\Omega_{0} \in \mathscr{F}$, такое, что $\mathbf{P}\left(\Omega_{0}\right)=1$ u

$$
\lim _{k \rightarrow+\infty} d\left(\varphi_{k}\left(\theta_{-k} \omega\right) u, \mathscr{K}_{\omega}\right)=0 \quad \text { для любых } \omega \in \Omega_{0}, u \in H .
$$

Тогда СДС $\varphi_{k}(\omega)$ обладает случайным аттрактором $\mathscr{A}_{\omega}$, измеримым относительно прошлого $\mathscr{F}^{-}$. 
(ii) Если $\mathfrak{M} \in \mathscr{I}_{\varphi, \mathscr{F}^{-}}-$марковская инвариантная мера, то ее дезинтеграиия $\mu_{\omega}$ сосредоточена в кажсом случайном аттракторе $\mathscr{A}_{\omega}^{\prime}$, m. е. $\mu_{\omega}\left(\mathscr{A}_{\omega}^{\prime}\right)=1$ n. $\mathcal{H}$.

НАБРОСОК ДОКАЗАТЕЛЬСТвА. (i) Как показано в [7], в условиях доказываемого предложения СДС $\varphi_{k}(\omega)$ обладает случайным точечным аттрактором $\mathscr{A}_{\omega}$ в смысле сходимости п. н. Так как преобразования $\theta_{k}$ сохраняют меру, то $\mathscr{A}_{\omega}$ удовлетворяет (1.5). В силу конструкции этого множества оно измеримо относительно прошлого.

(ii) Пусть $\mathscr{A}_{\omega}^{\prime}$ - произвольный случайный аттрактор. Для доказательства того, что дезинтеграция инвариантной меры $\mathfrak{M} \in \mathscr{I}_{\varphi, \mathscr{F}}$ сосредоточена в $\mathscr{A}_{\omega}^{\prime}$, достаточно заметить, что (1.4) влечет за собой сходимость п. н. (1.5) вдоль некоторой подпоследовательности $k=k_{n}$, и повторить рассуждение из $[7$, Theorem 4.3].

\section{§2. Основные результаты}

Как и выше, через $(\Omega, \mathscr{F}, \mathbf{P})$ обозначается вероятностное пространство и через $H$ - польское пространство с метрикой $d$, наделенное борелевской сигмаалгеброй $\mathscr{B}_{H}$. Пусть $\left\{\varphi_{k}(\omega)\right\}$ - СДС на $H$ над группой сохраняющих меру преобразований $\theta_{k}$. Рассмотрим две гипотезы:

Условие 2.1. Перемешивание. Марковское семейство $\left\{\varphi_{k}(\omega) u\right\}$ является перемешивающим в следующем смысле: оно имеет единственную стационарную меру $\mu$ и для любой функции $f \in L(H)$ и произвольной начальной точки $u \in H$

$$
\mathfrak{P}_{k} f(u)=\mathbf{E} f\left(\varphi_{k}(\cdot) u\right) \rightarrow(\mu, f)=\int_{H} f(u) \mu(d u) \quad \text { при } k \rightarrow \infty .
$$

УСловие 2.2. Компактностъ. Найдется случайное компактное множество, притягивающее траектории системы $\varphi_{k}(\omega)$ (в смысле предложения 1.6). Более того, для любых $u \in H$ и $\varepsilon>0$ найдется $\Omega_{\varepsilon} \in \mathscr{F}$, компактное множество $K_{\varepsilon} \subset H$ и целое число $k_{\varepsilon}=k_{\varepsilon}(u) \geqslant 1$, такие, что $\mathbf{P}\left(\Omega_{\varepsilon}\right) \geqslant 1-\varepsilon$ и

$$
\varphi_{k}\left(\theta_{-k} \omega\right) u \in K_{\varepsilon} \quad \text { для } \omega \in \Omega_{\varepsilon}, k \geqslant k_{\varepsilon} .
$$

Пусть $\mu \in \mathscr{S}_{\varphi}$ - единственная стационарная мера для марковской полугруппы $\mathfrak{P}_{k}^{*}$ и $\mathfrak{M} \in \mathscr{I}_{\varphi, \mathscr{F}^{-}}-$соответствующая марковская мера, инвариантная относительно косого произведения $\Theta_{k}$ (см. предложение 1.4).

ТЕОрема 2.3. Предположим, что выполнено условие 2.1. Тогда для любой бункиии $F(\omega, u) \in \mathbb{L}\left(H, \mathscr{F}^{-}\right)$мъи имеем

$$
\mathbf{E} F\left(\Theta_{k}(\cdot, u)\right) \rightarrow(\mathfrak{M}, F)=\int_{\Omega} \int_{H} F(\omega, u) \mu_{\omega}(d u) \mathbf{P}(d \omega) \quad \text { npu } k \rightarrow \infty,
$$

где $u \in H-$ произвольная начальная точка.

Теперь мы обсудим соотношения между инвариантными мерами и случайными аттракторами. Обозначим через $\mu_{\omega}$ дезинтеграцию меры $\mathfrak{M}$ и положим

$$
\mathscr{A}_{\omega}= \begin{cases}\operatorname{supp} \mu_{\omega}, & \omega \in \Omega_{0}, \\ H, & \omega \notin \Omega_{0},\end{cases}
$$


где $\Omega_{0} \in \mathscr{F}$ - множество полной меры, на котором существует предел (1.3). В силу следствия 1.6 .5 из [3] $\mathscr{A}_{\omega}$ является случайным замкнутым множеством. Более того, в силу (1.3) это множество измеримо по отношению к прошлоMy $\mathscr{F}^{-}$.

Теорема 2.4. Предположим, что выполнены условия 2.1 и 2.2. Тогда $\mathscr{A}_{\omega}$ является минимальным случайным точечным аттрактором.

Доказательства приведенных выше теорем даны в §3. Сейчас мы обсудим один класс СДС, удовлетворяющих условиям 2.1 и 2.2.

Пример 2.5. Случайно возмущенные динамические системы. Пусть $H-$ гильбертово пространство с нормой $|\cdot|$ и ортонормированным базисом $\left\{e_{j}\right\}$, и пусть $\mathrm{P}_{N}$ - ортогональный проектор на подпространство $H_{N} \subset H$, порожденное векторами $e_{1}, \ldots, e_{N}$. Предположим, что непрерывный оператор $S: H \rightarrow H$ удовлетворяет следующим двум условиям:

$$
\begin{gathered}
|S(u)| \leqslant q|u| \text { для } u \in H, \\
\left|\mathrm{P}_{N}(S(u)-S(v))\right| \leqslant \frac{1}{2}|u-v| \quad \text { для }|u| \vee|v| \leqslant R,
\end{gathered}
$$

где $q<1$ - константа, не зависящая от $u, R>0$ - произвольная константа и $N \geqslant 1$ - целое число, зависящее только от $R$. Рассмотрим СДС, порожденную уравнением

$$
u_{k}=S\left(u_{k-1}\right)+\eta_{k}
$$

где $k \in \mathbb{Z}$ и $\eta_{k}$ - последовательность независимых одинаково распределенных $H$-значных случайных величин. Если распределение $\chi$ случайных величин $\eta_{k}$ имеет компактный носитель, то условие 2.2 выполнено. Если вдобавок мера $\chi$ невырожденна (в смысле [13]), то марковское семейство, отвечающее $(2.7)$, является системой перемешивающего типа. Таким образом, при наложенных выше условиях носитель единственной инвариантной меры является случайным точечным аттрактором. Заметим, что уравнение (2.7), отвечающее системе Навье-Стокса, возмущенной случайной толчковой силой (см. ниже), удовлетворяет (2.5) и (2.6); см. [13].

Условия 2.1 и 2.2 выполнены также для большого класса неограниченных случайных толчковых сил $\eta_{k}$ (см. (2.7)). Мы не будем задерживаться на обсуждении этого случая.

ЗАмЕчАниЕ 2.6. Теоремы 2.3 и 2.4 остаются верными для СДС $\varphi_{t}(\omega)$ с непрерывным временем $t \geqslant 0$. В этом случае мы предполагаем, что отображение $\varphi_{t}(\omega) u$ непрерывно по $(t, u)$ для любого фиксированного $\omega \in \Omega$ и что условия 2.1 и 2.2 выполнены с $k$, замененным на $t$. Переформулировки приведенных выше результатов и их доказательств на случай непрерывного времени достаточно очевидны, и мы не будем формулировать соответствующих утверждений.

\section{§3. Доказательства}

3.1. Доказательство теоремы 2.3. Шаг 1. Предположим сначала, что $F(\omega, u) \in \mathbb{L}\left(H, \mathscr{F}_{[-\ell, 0]}\right)$, где $\ell \geqslant 0$ - целое число. Так как преобразования $\theta_{k}$ сохраняют меру, то для любого $m \geqslant 1$ имеем

$$
\begin{aligned}
p_{k}(u): & =\mathbf{E} F\left(\theta_{k} \omega, \varphi_{k}(\omega) u\right)=\mathbf{E} F\left(\omega, \varphi_{k}\left(\theta_{-k} \omega\right) u\right) \\
& =\mathbf{E} \mathbf{E}\left\{F\left(\omega, \varphi_{k}\left(\theta_{-k} \omega\right) u\right) \mid \mathscr{F}_{[1-m, 0]}\right\} .
\end{aligned}
$$


В силу свойства коцикла (см. (1.1))

$$
\varphi_{k}\left(\theta_{-k} \omega\right)=\varphi_{m}\left(\theta_{-m} \omega\right) \varphi_{k-m}\left(\theta_{-k} \omega\right), \quad m \leqslant k .
$$

Следовательно, положив $F_{m}(\omega, u)=F\left(\omega, \varphi_{m}\left(\theta_{-m} \omega\right) u\right)$, для любого $m \leqslant k$ имеeM

$$
p_{k}(u)=\mathbf{E} \mathbf{E}\left\{F_{m}\left(\omega, \varphi_{k-m}\left(\theta_{-k} \omega\right) u\right) \mid \mathscr{F}_{[1-m, 0]}\right\} .
$$

Заметим теперь, что $F_{m} \in \mathbb{L}\left(H, \mathscr{F}_{[1-m, 0]}\right)$, если $m \geqslant \ell+1$. Так как отображение $\varphi_{k-m}\left(\theta_{-k} \omega\right) u$ измеримо по отношению к сигма-алгебре $\mathscr{F}_{[1-k,-m]}$ и так как сигма-алгебры $\mathscr{F}_{[1-m, 0]}$ и $\mathscr{F}_{[1-k,-m]}$ независимы, то из (3.1) следует, что

$$
p_{k}(u)=\mathbf{E} \mathbf{E}^{\prime}\left\{F_{m}\left(\omega, \varphi_{k-m}\left(\theta_{-k} \omega^{\prime}\right) u\right)\right\}=\mathbf{E}\left(\mathfrak{P}_{k-m} F_{m}\right)(\omega, u),
$$

где $\ell+1 \leqslant m \leqslant k$ и $\mathbf{E}^{\prime}$ означает математическое ожидание по отношению к $\omega^{\prime}$. В силу условия 2.1 и теоремы Лебега для любого $m \geqslant \ell+1$ правая часть соотношения $(3.2)$ сходится к $\mathbf{E}\left(\mu, F_{m}(\omega, \cdot)\right)$ при $k \rightarrow+\infty$. Учитывая определение функции $F_{m}$, мы видим, что

$$
\left(\mu, F_{m}(\omega, \cdot)\right)=\left(\varphi_{m}\left(\theta_{-m} \omega\right) \mu, F(\omega, \cdot)\right) \rightarrow\left(\mu_{\omega}, F(\omega, \cdot)\right) \quad \text { при } m \rightarrow \infty,
$$

где было использовано предложение 1.4. Таким образом,

$$
\lim _{k \rightarrow+\infty} p_{k}(u)=\mathbf{E}\left(\mu_{\omega}, F(\omega, \cdot)\right)
$$

т. е. равенство (2.3) доказано.

Шаг 2. Теперь докажем, что равенство (2.3) выполнено для функций вида $F(\omega, u)=f(u) g(\omega)$, где $f \in L(H)$ и $g$ - ограниченная $\mathscr{F}^{-}$-измеримая функция. Для этого мы воспользуемся теоремой о монотонном классе (см. [2, теорема 3.3]). Зафиксируем произвольную функцию $f \in L(H)$ и обозначим через $\mathscr{H}$ множество ограниченных $\mathscr{F}^{-}$-измеримых функций $g$, для которых (2.3) выполнено при $F=f g$. Понятно, что $\mathscr{H}$ - линейное пространство, содержащее постоянные функции. Более того, как было показано на шаге 1 , оно содержит все ограниченные функции, измеримые по отношению к сигма-алгебре $\mathscr{F}[-\ell, 0]$ при некотором $\ell \geqslant 0$. Так как объединение всех этих сигма-алгебр порождает $\mathscr{F}^{-}$, то требуемое утверждение будет доказано, как только мы проверим следующее свойство: если $g_{n} \in \mathscr{H}$ - возрастающая последовательность неотрицательных функций, таких, что $g=\sup g_{n}$ является ограниченной функцией, то $g \in \mathscr{H}$.

Пусть $\left\{g_{n}\right\} \subset \mathscr{H}$ - последовательность, такая же, как выше. Без потери общности мы можем считать, что $0 \leqslant g, g_{n} \leqslant 1$. По теореме Егорова для любого $\varepsilon>0$ найдется $\Omega_{\varepsilon} \in \mathscr{F}$, такое, что $\mathbf{P}\left(\Omega_{\varepsilon}\right) \geqslant 1-\varepsilon$ и

$$
\lim _{k \rightarrow+\infty} \sup _{\omega \in \Omega_{\varepsilon}}\left|g_{n}(\omega)-g(\omega)\right|=0 .
$$

Следовательно, для любого $\varepsilon>0$ найдется целое число $n_{\varepsilon} \geqslant 1$, такое, что $n_{\varepsilon} \rightarrow+\infty$, когда $\varepsilon \rightarrow 0$, и

$$
g_{n_{\varepsilon}}(\omega) \leqslant g(\omega) \leqslant g_{n_{\varepsilon}}(\omega)+\varepsilon+I_{\Omega_{\varepsilon}^{c}}(\omega) \quad \text { для всех } \omega \in \Omega .
$$

Умножая это неравенство на $f\left(\varphi_{k}\left(\theta_{-k} \omega\right) u\right)$, беря математическое ожидание и переходя к пределу при $k \rightarrow+\infty$ с использованием оценки $\mathbf{P}\left(\Omega_{\varepsilon}^{c}\right) \leqslant \varepsilon$, мы 
получаем, что

$$
\begin{aligned}
\mathbf{E}\left\{\left(\mu_{\omega}, f\right) g_{n_{\varepsilon}}(\omega)\right\} & \leqslant \liminf _{k \rightarrow+\infty} \mathbf{E}\left\{f\left(\varphi_{k}\left(\theta_{-k} \omega\right) u\right) g(\omega)\right\} \\
& \leqslant \limsup _{k \rightarrow+\infty} \mathbf{E}\left\{f\left(\varphi_{k}\left(\theta_{-k} \omega\right) u\right) g(\omega)\right\} \leqslant \mathbf{E}\left\{\left(\mu_{\omega}, f\right) g_{n_{\varepsilon}}(\omega)\right\}+2 \varepsilon .
\end{aligned}
$$

Так как $\varepsilon>0$ произвольно и $\mathbf{E}\left\{\left(\mu_{\omega}, f\right) g_{n_{\varepsilon}}(\omega)\right\} \rightarrow \mathbf{E}\left\{\left(\mu_{\omega}, f\right) g(\omega)\right\}$ при $\varepsilon \rightarrow 0$ (в силу теоремы о монотонной сходимости), то

$$
\mathbf{E}\left\{f\left(\varphi_{k}(\omega) u\right) g\left(\theta_{k} \omega\right)\right\}=\mathbf{E}\left\{f\left(\varphi_{k}\left(\theta_{-k} \omega\right) u\right) g(\omega)\right\} \underset{k \rightarrow+\infty}{\longrightarrow} \mathbf{E}\left\{\left(\mu_{\omega}, f\right) g(\omega)\right\} .
$$

Таким образом, $g \in \mathscr{H}$. Это завершает доказательство (2.3) в случае, когда $F(\omega, u)=f(u) g(\omega)$.

Шаг 3. Теперь рассмотрим общий случай. Пусть $F \in \mathbb{L}(H)-$ произвольная функция, такая, что $\|F(\omega, \cdot)\|_{L(H)} \leqslant 1$ при п. в. $\omega \in \Omega$. Для любых $u \in H$ и $\varepsilon>0$ выберем целое число $k_{\varepsilon}(u) \geqslant 1$ и множества $\Omega_{\varepsilon} \in \mathscr{F}, K_{\varepsilon} \Subset H$, для которых выполнено (2.2). В силу теоремы Асколи-Арцела единичный шар $B_{\varepsilon}=\left\{f \in L\left(K_{\varepsilon}\right):\|f\|_{L(H)} \leqslant 1\right\}$ компактен в пространстве $C_{b}\left(K_{\varepsilon}\right)$. Следовательно, этот шар имеет конечную $\varepsilon$-сеть $\left\{h_{j}\right\} \subset B_{\varepsilon}$. Поэтому он может быть покрыт непересекающимися борелевскими множествами $U_{j} \ni h_{j}, j=1, \ldots, N$, диаметры которых не превосходят $2 \varepsilon$. Обозначим через $f_{j} \in L(H)$ произвольные продолжения функций $h_{j}$ на $H$, такие, что $\left\|f_{j}\right\|_{L(H)} \leqslant 2$. Например, можно выбрать

$$
f_{j}(u)=\inf _{v \in K_{\varepsilon}}\left(h_{j}(v)+d(u, v) \wedge 1\right) .
$$

Рассмотрим следующую аппроксимацию функции $F$ :

$$
G_{\varepsilon}(\omega, u)=\sum_{j=1}^{N} f_{j}(u) g_{j}(\omega), \quad g_{j}(\omega)=I_{U_{j}}\left(F_{K_{\varepsilon}}(\omega, \cdot)\right),
$$

где $F_{K_{\varepsilon}}(\omega, u)$ - сужение функции $F$ на $\Omega \times K_{\varepsilon}$. Так как лишь одна из функций $g_{j}$ может быть ненулевой, то $\left\|G_{\varepsilon}(\omega, \cdot)\right\|_{\infty} \leqslant 2$. Таким образом, для любого $u \in H$ и для почти любого $\omega \in \Omega$ мы имеем

$$
\begin{aligned}
\left|G_{\varepsilon}(\omega, u)-F(\omega, u)\right| & \leqslant 2 \varepsilon+I_{K_{\varepsilon}^{c}}(u)\left(\left\|G_{\varepsilon}(\omega, \cdot)\right\|_{\infty}+\|F(\omega, \cdot)\|_{\infty}\right) \\
& \leqslant 2 \varepsilon+3 I_{K_{\varepsilon}^{c}}(u),
\end{aligned}
$$

где мы воспользовались тем, что $\|F(\omega, \cdot)\|_{\infty} \leqslant 1$. Положим

$$
p_{k}(u)=\mathbf{E} F\left(\theta_{k} \omega, \varphi_{k}(\omega) u\right), \quad p_{k}(u, \varepsilon)=\mathbf{E} G_{\varepsilon}\left(\theta_{k} \omega, \varphi_{k}(\omega) u\right) .
$$

Тогда

$$
\left|p_{k}(u)-(\mathfrak{M}, F)\right| \leqslant\left|p_{k}(u)-p_{k}(u, \varepsilon)\right|+\left|p_{k}(u, \varepsilon)-\left(\mathfrak{M}, G_{\varepsilon}\right)\right|+\left|\left(\mathfrak{M}, G_{\varepsilon}-F\right)\right|
$$

Оценим каждое слагаемое в правой части неравенства (3.4). В силу (2.2) и (3.3) для $k \geqslant k_{\varepsilon}(u)$ мы имеем

$$
\begin{aligned}
\left|p_{k}(u)-p_{k}(u, \varepsilon)\right| & \leqslant\left|\mathbf{E}\left\{F\left(\omega, \varphi_{k}\left(\theta_{-k} \omega\right) u\right)-G_{\varepsilon}\left(\omega, \varphi_{k}\left(\theta_{-k} \omega\right) u\right)\right\}\right| \\
& \left.\leqslant 2 \varepsilon+3 \mathbf{P}\left\{\varphi_{k}\left(\theta_{-k} \omega\right) u\right) \notin K_{\varepsilon}\right\} \leqslant 2 \varepsilon+3 \mathbf{P}\left(\Omega_{\varepsilon}^{c}\right) \leqslant 5 \varepsilon .
\end{aligned}
$$

Далее, так как функции $g_{j}$ являются $\mathscr{F}^{-}$-измеримыми, то в силу шага 2

$$
p_{k}(u, \varepsilon) \rightarrow\left(\mathfrak{M}, G_{\varepsilon}\right) \quad \text { при } k \rightarrow+\infty
$$


для любого $\varepsilon>0$. Наконец, неравенство (3.3) влечет за собой неравенство

$$
\left|\left(\mathfrak{M}, G_{\varepsilon}-F\right)\right| \leqslant 2 \varepsilon+3\left(\mathfrak{M}, I_{K_{\varepsilon}^{c}}\right)=2 \varepsilon+3 \mu\left(K_{\varepsilon}^{c}\right) .
$$

Так как $\varepsilon>0$ произвольно, то в силу неравенств (3.4)-(3.7) для доказательства (2.3) остается установить, что $\mu\left(K_{\varepsilon}^{c}\right) \rightarrow 0$ при $\varepsilon \rightarrow 0$.

С этой целью мы заметим, что

$$
\mu\left(K_{\varepsilon}^{c}\right)=\int_{H} \mathbf{P}\left\{\varphi_{k}(\omega) u \notin K_{\varepsilon}\right\} \mu(d u) .
$$

Из условия 2.1 следует, что для любого фиксированного $u \in H$

$$
\limsup _{k \rightarrow+\infty} \mathbf{P}\left\{\varphi_{k}(\omega) u \notin K_{\varepsilon}\right\}=\limsup _{k \rightarrow+\infty} \mathbf{P}\left\{\varphi_{k}\left(\theta_{-k} \omega\right) u \notin K_{\varepsilon}\right\} \leqslant \varepsilon .
$$

Переходя к пределу в $(3.8)$ при $k \rightarrow+\infty$, получаем, что $\mu\left(K_{\varepsilon}^{c}\right) \leqslant \varepsilon$ для любого $\varepsilon>0$. Это завершает доказательство теоремы 2.3.

3.2. Доказательство теоремы 2.4. Покажем сначала, что случайное компактное множество $\mathscr{A}_{\omega}$ является случайным аттрактором. Зафиксируем произвольное $\delta \in(0,1)$ и рассмотрим функцию

$$
F(\omega, u)=1-\frac{d\left(u, \mathscr{A}_{\omega}\right)}{\delta} \wedge 1, \quad u \in H, \omega \in \Omega .
$$

Мы утверждаем, что $F \in \mathbb{L}\left(H, \mathscr{F}^{-}\right)$. Действительно, в силу определения функция $F$ ограничена и

$$
|F(\omega, u)-F(\omega, v)| \leqslant \frac{d(u, v)}{\delta} \quad \text { для всех } u, v \in H, \omega \in \Omega .
$$

Поэтому $F$ удовлетворяет условию (0.6). Так как случайное компактное множество $\mathscr{A}_{\omega}$ измеримо по отношению к $\mathscr{F}^{-}$, то случайная величина $\omega \rightarrow d\left(u, \mathscr{A}_{\omega}\right)$ измерима по отношению к $\mathscr{F}^{-}$для любого $u \in H$ (см. [3, Sec. 6.1]). Тем самым требуемые свойства функции $F$ установлены.

Так как $F(\omega, u)=1$ для $u \in \mathscr{A}_{\omega}$, то $(\mathfrak{M}, F)=1$. Поэтому, применяя теорему 2.3, мы устанавливаем, что

$$
\mathbf{E} F\left(\theta_{k} \omega, \varphi_{k}(\omega) u\right)=1-\mathbf{E}\left(\frac{d\left(\varphi_{k}(\omega) u, \mathscr{A}_{\theta_{k} \omega}\right)}{\delta} \wedge 1\right) \rightarrow(\mathfrak{M}, F)=1,
$$

T. e.

$$
p_{k}(u):=\mathbf{E}\left(\frac{d\left(\varphi_{k}(\omega) u, \mathscr{A}_{\theta_{k} \omega}\right)}{\delta} \wedge 1\right) \rightarrow 0 .
$$

Заметим теперь, что по неравенству Чебышева

$$
\mathbf{P}\left\{d\left(\varphi_{k}(\omega) u, \mathscr{A}_{\theta_{k} \omega}\right)>\delta\right\} \leqslant \frac{p_{k}(u)}{\delta} .
$$

В силу (3.9) правая часть этого неравенства стремится к нулю при $k \rightarrow+\infty$. Это завершает доказательство того, что множество $\mathscr{A}_{\omega}$ является случайным аттрактором.

Чтобы доказать, что $\mathscr{A}_{\omega}$ является минимальным случайным аттрактором, достаточно заметить, что в силу предложения 1.6 инвариантная мера $\mathfrak{M} \in$ $\mathscr{I}_{\mathbf{P}, \mathscr{F}-}$ сосредоточена в любом случайном аттракторе $\mathscr{A}_{\omega}^{\prime}$. Таким образом, $\operatorname{supp} \mu_{\omega} \subset \mathscr{A}_{\omega}^{\prime}$ для п. в. $\omega \in \Omega$. 


\section{§4. Система Навье-Стокса}

В этом параграфе мы рассматриваем двумерную систему Навье-Стокса (НC)

$$
\dot{u}-\nu \Delta u+(u, \nabla) u+\nabla p=\eta(t, x), \quad \operatorname{div} u=0 .
$$

Пространственная переменная $x$ принадлежит либо гладкой ограниченной области $D$ и тогда предполагается выполненным краевое условие Дирихле $\left.u\right|_{\partial D}=0$, либо двумерному тору $\mathbb{T}^{2}=\mathbb{R}^{2} / 2 \pi \mathbb{Z}^{2}$ и тогда предполагается, что $\int u d x=\int \eta d x \equiv 0$. Нас интересует эволюция во времени поля скорости $u$ (не давления $p$ ). Соответственно мы заменяем силу $\eta$ ее соленоидальной компонентой (пренебрегая градиентной компонентой) и всюду ниже считаем, что

$$
\operatorname{div} \eta=0 .
$$

Сначала мы рассмотрим случай, когда $\eta$ является случайной толчковой силой

$$
\eta(t, x)=\sum_{k \in \mathbb{Z}} \delta(t-k) \eta_{k}(x),
$$

где $\eta_{k}$ - независимые равнораспределенные случайные поля, как в $[12,13]$. Через $H$ обозначим гильбертово пространство, образованное бездивергентными векторными полями на рассматриваемой области, удовлетворяющими краевым условиям в обычном смысле (например, см. [1]). Мы нормализуем решения $u(t, x)$ уравнения (4.1), (4.2), трактуемые как случайные кривые в пространстве $H$, и считаем их непрерывными справа. Тогда, измеряя значения решений в целые моменты времени $t=k \in \mathbb{Z}_{+}$и полагая $u_{k}=u(k, \cdot)$, мы получаем уравнение

$$
u_{k}=S\left(u_{k-1}\right)+\eta_{k} .
$$

Здесь $S$ - оператор сдвига за единичное время по траекториям свободной системы (4.1) (с $\eta \equiv 0)$; см. [13]. Пусть $\varphi_{k}, k \geqslant 0,-$ СДС, порожденная уравнением $(4.3)^{1)}$. Несложная проверка показывает, что эта система обладает свойством компактности (cp. [12, Sec. 2.2.1]). Более того, если распределение толчков $\eta_{k}$ удовлетворяет неограничительному условию невырожденности, приведенному в [13], то соответствующая цепь Маркова в $H$ имеет единственную стационарную меру $\mu$ и выполнено условие (0.4). Следовательно, применима теорема 2.4 и мы получаем следующий результат:

Теорема 4.1. Если случайная сила (4.2) удовлетворяет приведенным выше условиям, то носитель $\mu_{\omega}$ марковской дезинтеграции соответствующей единственной стационарной меры $\mu$ является минимальным случайным аттрактором для СДС (4.3). Более того, найдется детерминистская константа $D=D_{\nu}$, такая, что хаусдорфова размерность множества $\mathscr{A}_{\omega}$ не превосxодит $D$ при п. в. $\omega$.

В замечании 2.6 мы отмечаем, что утверждение теоремы 2.4 остается в силе для некоторого класса СДС с непрерывным временем $t \geqslant 0$. Этот класс

1) Отображения $\varphi_{k}, k \geqslant 0$, переводят $u \in H$ в решение $u_{k}$ уравнения (4.3), равное $u$ при $t=0$. 
содержит систему, описывающую двумерное уравнение Навье-Стокса (4.1), в которой сила $\eta$ как функция времени является белым шумом:

$$
\eta(t, x)=\frac{\partial}{\partial t} \zeta, \quad \zeta=\sum_{j=1}^{\infty} b_{j} \beta_{j}(t) e_{j}(x) .
$$

Здесь $\left\{e_{j}\right\}$ - это $L^{2}$-нормализованный тригонометрический базис пространства $H$, a $\left\{\beta_{j}, t \in \mathbb{R}\right\}$ - семейство независимых стандартных винеровских процессов. Предполагается, что вещественные коэффициенты $b_{j}$ убывают быстрее любой отрицательной степени:

$$
\left|b_{j}\right| \leqslant C_{m} j^{-m} \quad \text { для всех } j, m \geqslant 1,
$$

так что случайное поле $\eta(t, x)$ п. н. гладко по $x$. Обозначим через $\mathscr{H}$ пространство непрерывных кривых $\xi: \mathbb{R} \rightarrow H$, таких, что $\xi(0)=0$, и наделим его топологией равномерной сходимости на ограниченных интервалах. Пусть $\mathscr{B}-$ сигма-алгебра борелевских подмножеств пространства $\mathscr{H},\left\{\theta_{t}\right\}$ - группа канонических сдвигов пространства $\mathscr{H}, \theta_{t} \xi(s)=\xi(s+t)-\xi(t)$, и $\mathbf{P}$ - распределение процесса $\zeta$ в $\mathscr{H}$. Выберем $(\mathscr{H}, \mathscr{B}, \mathbf{P})$ в качестве основного вероятностного пространства $(\Omega, \mathscr{F}, \mathbf{P})$. Тогда НС-система $(4.1),(4.4)$ определяет СДС с непрерывным временем над группой сдвигов $\theta_{t}$ (см. [3]) и марковский процесс в $H$. Эта СДС удовлетворяет условию компактности, см. [5, Sec. 3.1]. Более того, как доказано в [14] (см. также $[4,11]$ ), найдется целое число $N=N_{\nu}$, такое, что если

$$
b_{j} \neq 0 \text { для } 1 \leqslant j \leqslant N,
$$

то соответствующий марковский процесс в $H$ имеет единственную стационарную меру $\mu$ и удовлетворяет (0.4). Мы получаем следующий результат:

ТЕОремА 4.2. Если выполнено условие (4.5), то уравнение (4.1), (4.4) имеет единственную стационарную меру $\mu$. Носитель $\mathscr{A}_{\omega}$ ее марковской дезинтеграиии $\mu_{\omega}$ определяет минимальный случайный аттрактор соответствующей СДС в пространстве Н. Более того, найдется детерминистская константа $D=D_{\nu}$, такая, что хаусдорфова размерность множества $\mathscr{A}_{\omega}$ не превосходum D nри n. в. $\omega$.

Тот факт, что множества $\operatorname{supp} \mu_{\omega}$ имеют конечную хаусдорфову размерность как в случае дискретного, так и в случае непрерывного времени, следует из общих результатов об оценке сверху на хаусдорфову размерность глобальных случайных аттракторов (см. [8-10]), так как последние содержат supp $\mu_{\omega}(\mathrm{cm}$. следствие 3.6 в [7]).

Рассмотрим косое произведение $\left\{\Theta_{k}\right\}$, отвечающее обсуждаемой СДС. Применяя версию теоремы 2.3 для систем с непрерывным временем, мы получаем следующий результат:

Теорема 4.3. Пусть $G$ - ограниченный измеримый функиионал на $H \times \mathscr{H}$, равномерно липшищевый по первой переменной и такой, что $G(u, \zeta(\cdot))$ зависит только от $\{\zeta(s), s \leqslant 0\}$. Пусть $u(t)$ является решением уравнения (4.1), (4.4), равным $u_{0}$ при $t=0$. Пусть выполнено предположение (4.5). Тогда следующая сходимость имеет место при любом $u_{0} \in H$ :

$$
\mathbf{E} G\left(u(t), \theta_{t} \zeta\right) \rightarrow \mathbf{E} \int_{H} F(v, \zeta) \mu_{\zeta}(d v) \quad \text { nрu } t \rightarrow \infty
$$




\section{ЛитеРАТУРА}

1. Лионс ЖК.-Л. Некоторые методы решения нелинейных краевых задач. Мир, М., 1972.

2. Ревюз Д. Цепи Маркова. Янус-К, М., 1997.

3. Arnold L. Random Dynamical Systems. Springer-Verlag, Berlin, 1998.

4. Bricmont J., Kupiainen A., Lefevere R. Exponential mixing for the 2D stochastic Navier-Stokes dynamics. Comm. Math. Phys., 230, No. 1, 87-132 (2002).

5. Crauel H., Debussche A., Flandoli F. Random attractors. J. Dynam. Diff. Eq., 9, No. 2, 307-341 (1997).

6. Crauel $H$. Markov measures for random dynamical systems. Stochastics Rep., 37, No. 3, 153-173 (1991).

7. Crauel $H$. Random point attractors versus random set attractors. J. London Math. Soc. (2), 63, No. 2, 413-427 (2001).

8. Chepyzhov V., Vishik M. A Hausdorff dimension estimate for kernel sections of nonautonomous evolution equations. Indiana Univ. Math. J., 42, No. 3, 1057-1076 (1993).

9. Chepyzhov V. V., Vishik M. I. Attractors for Equations of Mathematical Physics. Amer. Math. Soc. Coll. Publ., Vol. 49, Amer. Math. Soc., Providence, 2002.

10. Debussche A. Hausdorff dimension of a random invariant set. J. Math. Pures Appl. (9), 77, No. 10, 967-988 (1998).

11. E W., Mattingly J. C., Sinai Ya. G. Gibbsian dynamics and ergodicity for the stochastically forced Navier-Stokes equation. Comm. Math. Phys., 224, 83-106 (2001).

12. Kuksin S. B., Shirikyan A. Stochastic dissipative PDE's and Gibbs measures. Comm. Math. Phys., 213, 291-330 (2000).

13. Kuksin S. B., Shirikyan A. A coupling approach to randomly forced nonlinear PDE's. I. Comm. Math. Phys. 221, 351-366 (2001).

14. Kuksin S. B., Shirikyan A. Coupling approach to white-forced nonlinear PDE's. J. Math. Pures Appl., 81, 567-602 (2002).

15. Le Jan $Y$. Équilibre statistique pour les produits de difféomorphismes aléatoires indépendants. Ann. Inst. H. Poincaré Probab. Statist., 23, No. 1, 111-120 (1987).

16. Ledrappier F. Positivity of the exponent for stationary sequences of matrices, In: Lyapunov Exponents (Bremen, 1984), Lect. Notes in Math., Vol. 1186, Springer-Verlag, Berlin, 1986, pp. 56-73.

Хериот-Ватт Университет, Эдинбург, Великобритания Математический Институт РАН им. Стеклова

Поступило в редакцию e-mail: S.B.Kuksin@ma.hw.ac.uk

2 сентября 2002 г.

Хериот-Ватт Университет, Эдинбург, Великобритания e-mail: Armen.Shirikyan@math.u-psud.fr 